\title{
Analog Circuit Implementation and Full State Observation of Chua's Circuit
}

\author{
Hong Niu, Dongcheng Tan, Yongjun Wu \\ College of Electronic Information and Automation, Tianjin University of Science \& Technology, \\ 80 Mailbox, Tianjin University of Science \& Technology, No. 1038 Dagu Nanlu, Hexi District \\ Tianjin, 300222, China \\ E-mail:spots@163.com
}

\begin{abstract}
The inductance simulator and the nonlinear resistor, which are constructed by operational amplifiers and resistors, are applied to complete the analog circuit implementation of Chua's circuit as well as improve the accuracy of circuit parameters. The state variable $z$, which represents the product of the linear resistance and the current of the inductor, should be observed even if it is not an actual measurable physical quantity. It is found that $z$ can be obtained via scaling of the voltage of the resistor in the inductance simulator. The real chaotic curves generated from the analog Chua's circuit are displayed on the oscilloscope clearly. It demonstrates that the realization of Chua's circuit is correct.
\end{abstract}

Keywords: Chua's Circuit; Inductance Simulator; Analog Circuit Implementation; Scaling; Full State Observation

\section{Introduction}

Chua's circuit was introduced in 1983 by Leon Ong Chua. ${ }^{1}$ It is a three-dimensional autonomous electronic circuit that exhibits classic chaos behavior. Because Chua's circuit has rich chaotic dynamics and a simple circuit structure, it has been one of the best chaotic circuits for experimental observation and study. ${ }^{2-6}$

In Refs. 7-8, hybrid realizations of Chua's circuit, as well as the circuit topologies proposed for the nonlinear resistor and the inductor element, are presented. However, there is no study on how to observe the state variable $z$ which represents the product of the linear resistance and the current of the inductor. In this paper, Chua's circuit will be implemented by the inductance simulator and the piecewise linear resistor consisting of six resistors and two operational amplifiers, as shown in Ref. 8. Moreover, the state variable $z$ will be obtained via scaling of the voltage of the resistor in the inductance simulator to show the chaotic attractors of Chua's circuit completely.

\section{Chua's Circuit}

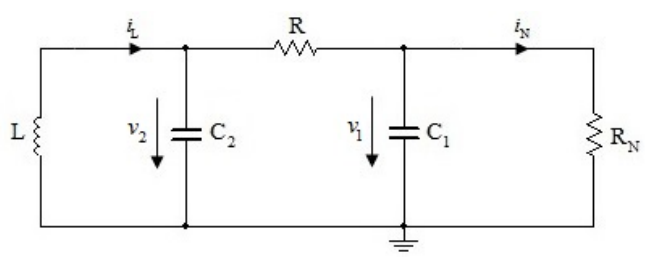

Fig. 1. Chua's circuit

Chua's circuit consists of two capacitors, one inductor, one linear resistor and one nonlinear resistor named Chua's diode, as shown in Fig. 1. The inductance, resistance and capacitances respectively are $\mathrm{L}=21.83$ $\mathrm{mH}, \mathrm{R}=1.875 \mathrm{k} \Omega, \mathrm{C}_{1}=10 \mathrm{nF}$ and $\mathrm{C}_{2}=100 \mathrm{nF}$. The volt-ampere characteristics of Chua's diode can be formulated as

$$
i_{\mathrm{N}}=\mathrm{G}_{\mathrm{b}} v_{1}+0.5\left(\mathrm{G}_{\mathrm{a}}-\mathrm{G}_{\mathrm{b}}\right)\left[\left|v_{1}+\mathrm{E}\right|-\left|v_{1}-\mathrm{E}\right|\right],
$$

where $\mathrm{E}=1.1 \mathrm{~V}, \mathrm{G}_{\mathrm{a}}=-0.7576 \mathrm{mS}$ and $\mathrm{G}_{\mathrm{b}}=-0.4091$ $\mathrm{mS}$. 
From Fig. 1, the state equation of Chua's circuit can be formulated as

$$
\begin{aligned}
\frac{\mathrm{d} v_{1}}{\mathrm{~d} t} & =\frac{1}{\mathrm{C}_{1}}\left(\frac{v_{2}-v_{1}}{\mathrm{R}}-i_{\mathrm{N}}\right), \\
\frac{\mathrm{d} v_{2}}{\mathrm{~d} t} & =\frac{1}{\mathrm{C}_{2}}\left(\frac{v_{1}-v_{2}}{\mathrm{R}}+i_{\mathrm{L}}\right), \\
\frac{\mathrm{d} i_{\mathrm{L}}}{\mathrm{d} t} & =-\frac{v_{2}}{\mathrm{~L}} .
\end{aligned}
$$

Let $x=v_{1}, y=v_{2}, z=\mathrm{R} i_{\mathrm{L}}$ and $t=\left(\mathrm{RC}_{2}\right) \tau$, and substitute them into Eq. (1). For convenience, abbreviate $\mathrm{d} \boldsymbol{x} / \mathrm{d} \tau$ to $\dot{\boldsymbol{x}}$. The dynamic equation of Chua's circuit is represented as

$$
\begin{aligned}
& \dot{x}=\alpha[y-x-h(x)], \\
& \dot{y}=x-y+z, \\
& \dot{z}=-\beta y,
\end{aligned}
$$

where $\alpha=\mathrm{C}_{2} / \mathrm{C}_{1}=10$ and $\beta=\left(\mathrm{R}^{2} \mathrm{C}_{2}\right) / \mathrm{L}=16.1$.

Compare Eq. (2) with Eq. (1), then,

$$
h(x)=m_{1} x+0.5\left(m_{0}-m_{1}\right)[|x+\mathrm{E}|-|x-\mathrm{E}|],
$$

where $m_{0}=\mathrm{RG}_{\mathrm{a}}=-1.4205$ and $m_{1}=\mathrm{RG}_{\mathrm{b}}=-0.7671$.

Let the initial values of the system (2) be $\left(x_{0}, y_{0}, z_{0}\right)$ $=(0.1,0.2,0.5)$, then the Lyapunov exponents are $\lambda_{1}=0.3193>0, \quad \lambda_{2}=-0.0001 \approx 0 \quad$ and $\quad \lambda_{3}=-3.1683<0$. It indicates that the system (2) is chaotic. The simulation phase portraits of Chua's circuit are shown in Fig. 3 (a1)-(a3).

\section{Implementation of Chua's Circuit}

\subsection{Inductance simulator}

Because the internal resistance of an ordinary inductor is too large and the inductance is difficult to alter, it is not suitable to put an ordinary inductor in a chaotic circuit which is very sensitive to the parameters. Hence, the inductance simulator should be used to construct Chua's circuit instead of an ordinary inductor. ${ }^{6-8}$ The equivalent circuit of the inductance simulator is shown in the left dashed box in Fig. 2, where $R_{1}=R_{2}=R_{3}=1$ $\mathrm{k} \Omega, \mathrm{R}_{4}=2.183 \mathrm{k} \Omega$ and $\mathrm{C}_{5}=10 \mathrm{nF}$. The operational amplifiers used in this paper are TL082CP and the supply voltages are $\pm 9 \mathrm{~V}$, so that the output saturation voltage is $8.3 \mathrm{~V}$. The inductance simulator has no equivalent internal resistance, and its inductance is easy to adjust.

Assume that the currents through the resistors $R_{1}$, $\mathrm{R}_{2}, \mathrm{R}_{3}, \mathrm{R}_{4}$ and the capacitor $\mathrm{C}_{5}$ respectively are $i_{1}, i_{2}, i_{3}$, $i_{4}$ and $i_{5}$, whose reference directions are all from top to bottom. When the operational amplifiers U1A and U1B are being operated in linear mode, it is obtained that

$$
\begin{aligned}
i_{2} & =i_{3}, \\
i_{4} & =i_{5}, \\
i_{1} \mathrm{R}_{1} & =-i_{2} \mathrm{R}_{2}, \\
i_{3} \mathrm{R}_{3} & =-i_{5} \frac{1}{\mathrm{j} \omega \mathrm{C}_{5}} .
\end{aligned}
$$

Thus, the relationship between the currents $i_{1}$ and $i_{4}$ is expressed as

$$
i_{4}=i_{1}\left(\mathrm{j} \omega \frac{\mathrm{R}_{1} \mathrm{R}_{3} \mathrm{C}_{5}}{\mathrm{R}_{2}}\right)
$$

According to the definition of two-terminal network impedance, the equivalent impedance of the inductance simulator is

$$
\mathrm{Z}=\frac{i_{4} \mathrm{R}_{4}}{i_{1}}=\mathrm{j} \omega \frac{\mathrm{R}_{1} \mathrm{R}_{3} \mathrm{R}_{4} \mathrm{C}_{5}}{\mathrm{R}_{2}} \rightarrow \mathrm{L}=21.83 \mathrm{mH}
$$

As a result, the desired inductance is obtainable by adjusting the resistance of the resistor $R_{4}$.

\subsection{Chua's diode}

Chua's diode consists of two operational amplifiers $\mathrm{U} 2 \mathrm{~A}, \mathrm{U} 2 \mathrm{~B}$ and six resistors, as shown in the right dotted box in Fig. 2. The resistances are $\mathrm{R}_{6}=\mathrm{R}_{7}=22 \mathrm{k} \Omega, \mathrm{R}_{8}=$ $3.3 \mathrm{k} \Omega, \mathrm{R}_{9}=\mathrm{R}_{10}=220 \Omega$ and $\mathrm{R}_{11}=2.2 \mathrm{k} \Omega$. The design procedure of this equivalent circuit can be found in detail in Ref. 6.

\subsection{Realization of Chua's circuit}

Chua's circuit is implemented by replacing the ordinary inductor and Chua's diode shown in Fig. 1 with the inductance simulator and the equivalent circuit of Chua's diode. The analog Chua's circuit is presented in Fig. 2. 


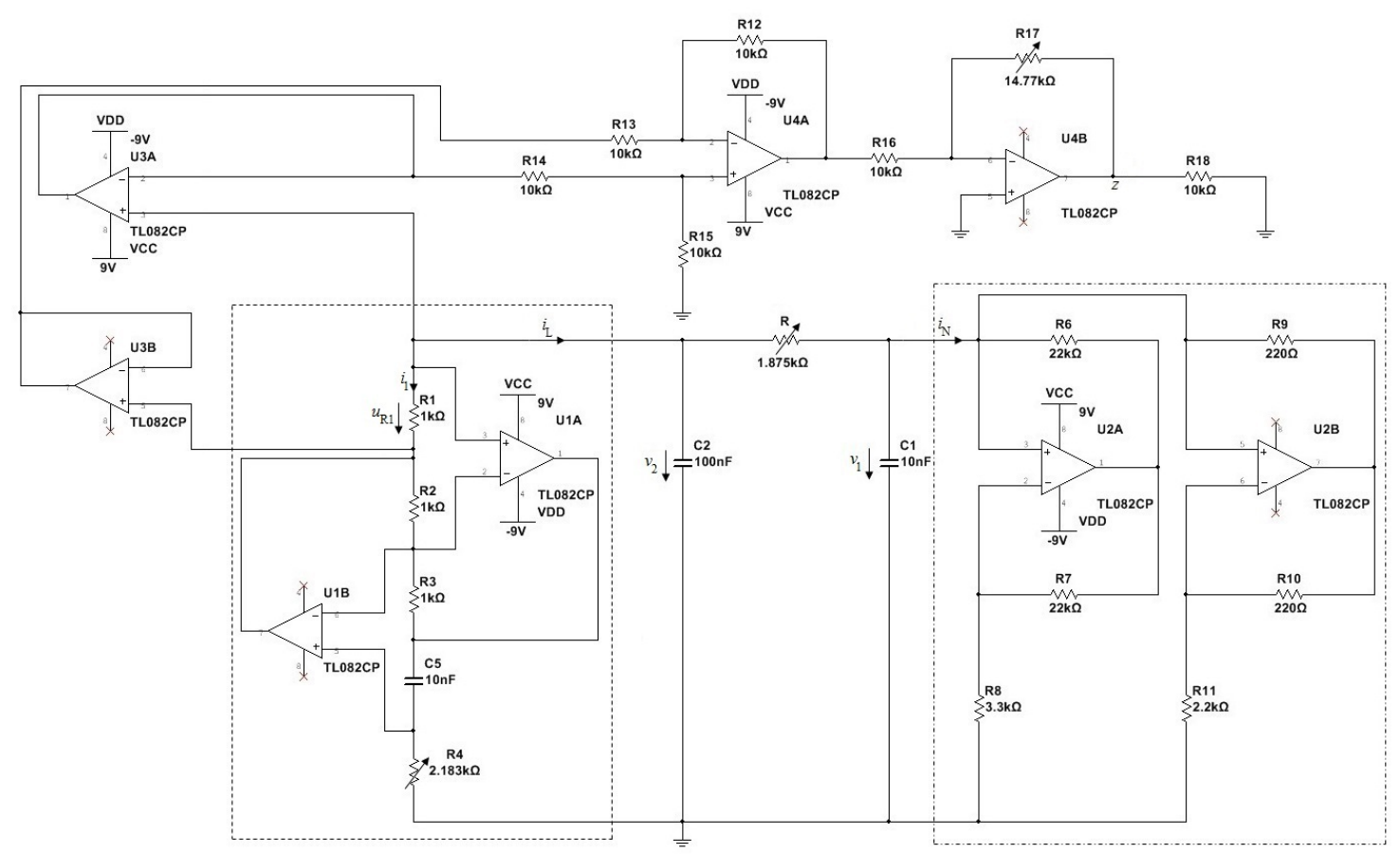

Fig. 2. Analog Chua's circuit

\section{Phase Portraits of Chua's Circuit}

\subsection{Observation of state variable $z$}

For the observation of all variables of the system (2), the state variable $z=\mathrm{R} i_{\mathrm{L}}$ should be observed even if it is not an actual measurable physical quantity in Chua's circuit. The observation circuit of $z$ consists of four operational amplifiers U3A, U3B, U4A, U4B and some related resistors, as shown in Fig. 2. The resistances are $\mathrm{R}_{12}=\mathrm{R}_{13}=\mathrm{R}_{14}=\mathrm{R}_{15}=\mathrm{R}_{16}=\mathrm{R}_{18}$ $=10 \mathrm{k} \Omega$, and the resistance of the resistor $R_{17}$ is variable.

The relationship between the voltage $u_{\mathrm{R} 1}$ and the state variable $z$ can be described as

$$
i_{\mathrm{L}}=-i_{1} \rightarrow z=\mathrm{R} i_{\mathrm{L}}=-\frac{\mathrm{R}}{\mathrm{R}_{1}} u_{\mathrm{R} 1}=-\frac{\mathrm{R}_{17}}{\mathrm{R}_{16}} u_{\mathrm{R} 1}
$$

Thus, the design procedure of the observation circuit can be divided into three parts. Firstly, U3A and U3B are two input voltage followers used to get the potentials of both ends of $R_{1}$. Secondly, U4A and the resistors $R_{12}-R_{15}$ constitute the differential input proportion operational circuit used to work out the voltage $u_{\mathrm{R} 1}$. Finally, the state variable $z$ can be obtained from the output of the inverse proportion operational circuit consisting of U4B and the resistors $R_{16}$ and $R_{17}$. Furthermore, the operational amplifiers $\mathrm{U} 3 \mathrm{~A}$ and $\mathrm{U} 3 \mathrm{~B}$ can also insulate the main Chua's circuit from the impact of the observation circuit.

It is found that some peak (valley) values of $z$ are larger than the output saturation voltage of the operational amplifier by solving the Eq. (2), such that the cut-off (saturation) distortion will occur when observing the experiment phase portraits of $x-z$ and $y-z$ on the oscilloscope. Hence, scaling of $z$ should be performed. Adjust the resistance of the resistor $R_{17}$ to $R_{17}=14.77$ $\mathrm{k} \Omega$, then the distortions will no longer occur. Consequently, the experiment value of $z$ is about 0.8 times its simulation value. For a convenient comparison, the simulation value of $z$ is adjusted to 0.8 times its original value in Fig. 3 (a2)-(a3).

\subsection{Comparison of simulation and experiment phase portraits}

The experiment phase portraits of Chua's circuit are shown in Fig. 3 (b1)-(b3). The scales of $x, y$ 
and $z$ are $2 \mathrm{~V} / \mathrm{Div}, 0.5 \mathrm{~V} / \mathrm{Div}$ and $2 \mathrm{~V} / \mathrm{Div}$, respectively. By comparing (b1)-(b3) with (a1)(a3) in Fig. 3, it can be confirmed that here is a good qualitative agreement between the numerical simulation and the experimental realization. It demonstrates that the realization of Chua's circuit is correct.

\section{Conclusions}

The hybrid analog circuit implementation of Chua's circuit, which exploits the circuit topologies for the inductor element and the nonlinear resistor, is proposed in this paper. The observation circuit for the directly non- measurable variable $z$ is also designed for comprehensive investigation of the chaotic attractors of Chua's circuit. It is necessary to observe all chaotic state variables, especially for full state chaos synchronization. For example, in synchronization physical test of two identical three-dimensional chaotic circuits, the assumption that all three states would synchronize as long as two of them synchronized may be incorrect, so that all of the states generated from the circuits should be investigated to verify the synchronization phenomena. Therefore, the study in this paper has some engineering significance.

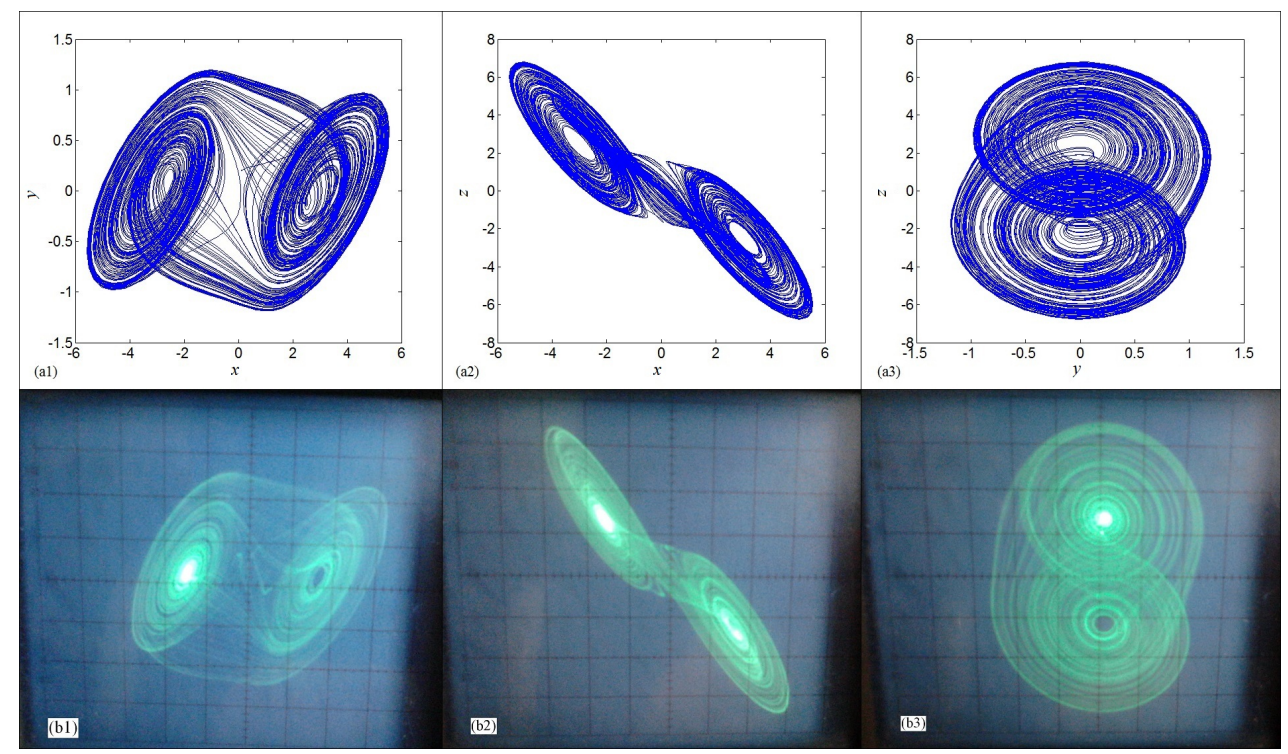

Fig. 3. Comparison of phase portraits: Simulation-Experiment: (a1)-(b1) $x-y$; (a2)-(b2) $x-z$; (a3)-(b3) $y-z$

\section{References}

1. T. Matsumoto, A chaotic attractor from Chua's circuit, IEEE Trans. on Circuits and Systems 31(12) (1984) $1055-1058$.

2. Z. Yang, J. Zhang, Y. Ma, Y. Bai and S. Ma, Design and realization of Chua's circuit based on current conveyers, Acta Physica Sinica 59(5) (2010) 3007-3016.

3. B. Bao, W. Hu, J. Xu, Z. Liu and L. Zou, Analysis and implementation of memristor chaotic circuit, Acta Physica Sinica 60(12) (2011) 63-70.

4. E. Lv and S. Huang, The equivalent circuit design of Chua's circuit and application thereof, Chinese Journal of Electron Devices 37(5) (2014) 891-895.

5. X. Zhang, H. Sun, J. Zhao, J. Liu, Y. Ma and T. Han, Equivalent circuit in function and topology to Chua's circuit and the design methods of these circuits, Acta Physica Sinica 63(20) (2014) 95-102.

6. S. Yu, Chaotic Systems and Chaotic Circuits: Principle, Design and Its Application in Communications (XiDian University Publishing House, Xi'an, 2011).

7. R. Kiliç, A comparative study on realization of Chua's circuit: Hybrid realizations of Chua's circuit combining the circuit topologies proposed for Chua's diode and inductor elements, International Journal of Bifurcation and Chaos 13(6) (2003) 1475-1493.

8. H. Liu, F. Jiang, B. Xia and L. Yue, The experiment study of multiscroll chaotic circuit based on a stimulated inductor circuit, Journal of Northeast Normal University (Natural Science Edition) 39(2) (2007) 55-59. 